\section{TRANSCRIPTIONAL ANALYSIS OF LEUKOCYTES FROM COVID CONVALESCENT DONORS REVEALS PERSISTENT ACTIVATION OF THE INNATE AND ADAPTIVE IMMUNE SYSTEM}

${ }^{1}$ Mallikarjuna Gedda, ${ }^{2}$ Patrick Danaher*, 'Lipei Saho, 'Martin Ongkeko, 'Leonard Chen, ${ }^{1}$ Mame Thioye Sall, ${ }^{1}$ Opal Reddy, ${ }^{2}$ Christina Bailey, ${ }^{2}$ Amy Wahba, ${ }^{2}$ Inna Dzekunova, ${ }^{1}$ Valeria De Giorgi, ${ }^{1}$ Robert Somerville, ${ }^{1}$ Jin Ping, ${ }^{1}$ Kamille West, ${ }^{1}$ Sandhya Panch, ${ }^{1}$ David Stroncek. ${ }^{1}$ National Institutes of Health, Bethesda, MD, USA; ${ }^{2}$ NanoString, Seattle, WA, USA

Background Coronavirus disease 2019 (COVID-19) results in robust but dysregulated acute immune response characterized by pro-inflammatory cytokine production and T-cell exhaustion, but little is known concerning immune response following recovery. We assessed immune function in convalescent plasma donors (CCD) who had recovered from COVID-19.

Methods The cellular immune response and $\mathrm{T}$-cell receptor (TCR) diversity in CCD was investigated using the nCounter host response and TCR diversity panels. 270 CCD and 40 healthy donor (HD) blood samples collected 11 to 193 days after diagnosis were analyzed. The CCD samples were from 162 donors, 69 donated more than once. All HD donated only once.
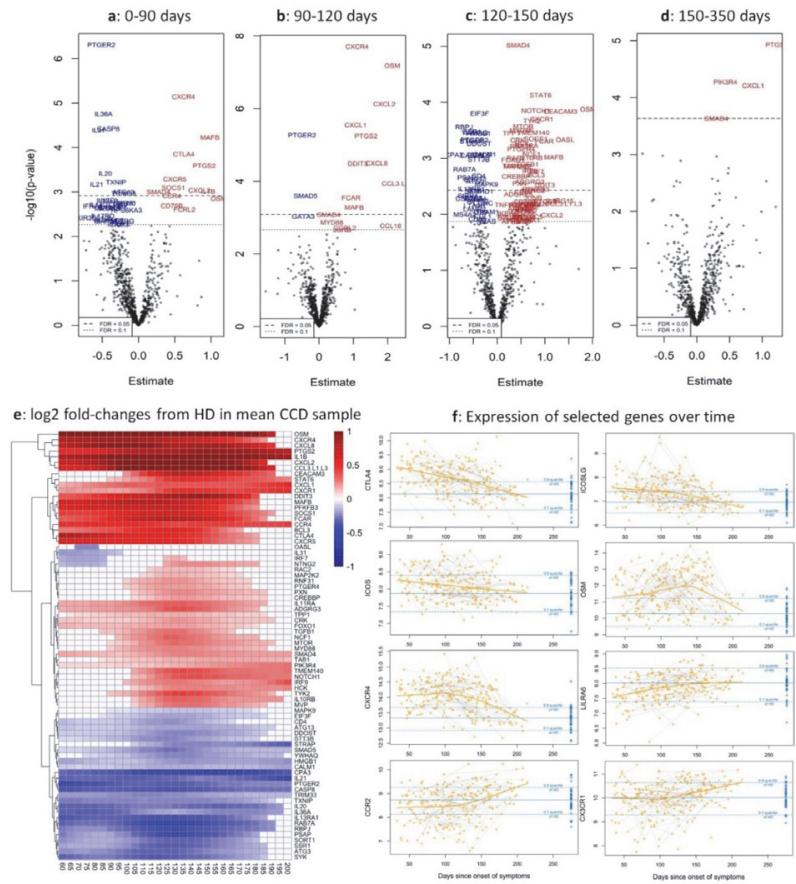

Abstract 953 Figure 1 Longitudinal trends in CCD gene expression. a-d: Differential expression results in HD vs. 4 time windows of CCD. Genes with FDR $<0.1$ are labeled; e: average CCD log2 fold-changes from HD over time. Color is only given for times where the Loess regression is different from the mean $H D$ with $p<0.05$; $f$ : longitudinal results for selected genes. Orange lines connect CCD samples over time. Blue lines show inner $95 \%$ quantiles of HD samples

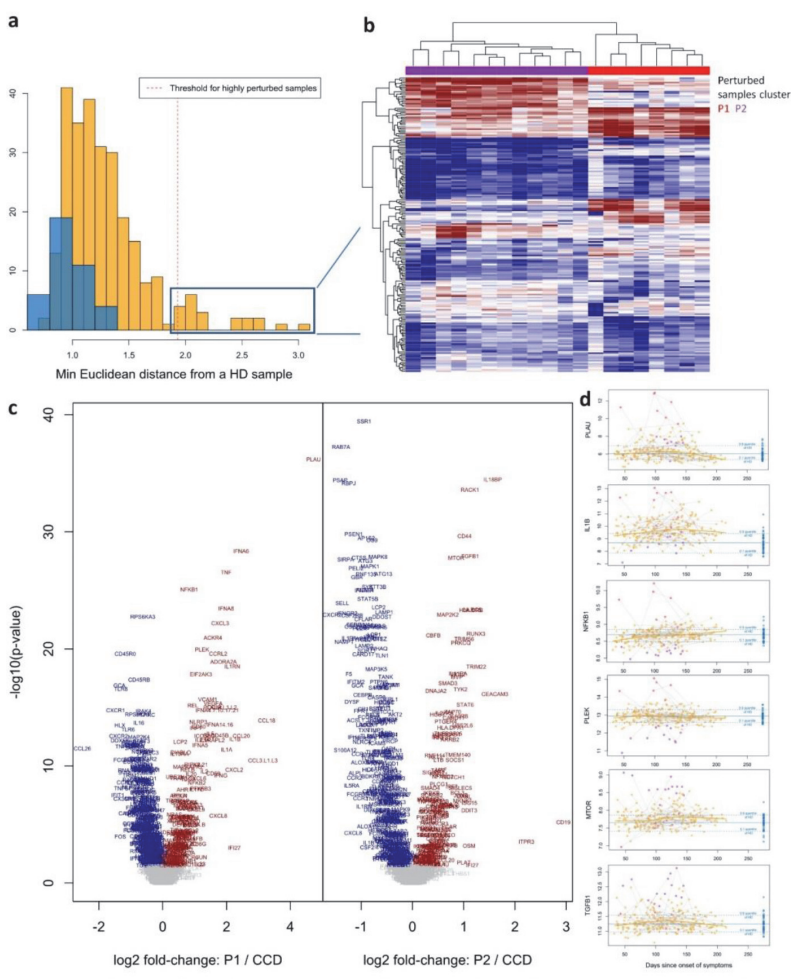

Abstract 953 Figure 2 CCD with more severe departure from HD gene expression. a: CCD samples (in orange) were scored for perturbation from the mean HD (in blue), and 21 highly perturbed sample subsets emerged; $\mathbf{b}$ : clustering of the 21 highly perturbed patients. The dendrogram was cut to define two groups. c: volcano plots comparing expression in P1 (left) and P2 (right) vs. CCD; d: longitudinal trends of selected genes perturbed in P1 and P2

Results Many genes were differentially expressed for months following infection. Analysis of samples collected 0 to 90 days post-diagnosis found that 19 of 773 genes were differentially expressed among CCD and HD (FDR $<0.05$ ) (figure 1a). At 90 to 120 days, 120 to 150 and $>150$ post-diagnosis, 13,58 and 4 genes were differentially expressed respectively (FDR < 0.05 ) (figures $1 \mathrm{~b}-\mathrm{d}$ ). At 120 to 150 days the differentially expressed genes included those in Treg differentiation, type III interferon signaling and chemokine signaling pathways. 76 genes were differently expressed at least once during the time windows described above. (Figure 1e). Among CCD, the expression of CTLA-4, ICOS, ICOSLG, OSM and CXCR4 were initially elevated but fell to HD levels at the end of the study period. The expression of LILRA6, CCR2 and CX3CR1 increased or remained elevated throughout (figure 1f).

A subset of samples departed notably from the average trend. The transcriptome of each CCD sample was scored by its similarity to the mean transcriptome of HD samples. This analysis revealed $21 \mathrm{CCD}$ samples from 19 unique donors 
were highly perturbed from HD samples (figure 2a). Among these highly perturbed samples $80 \%$ were collected $>90$ days post-diagnosis. The perturbed samples clustered into two groups, labelled P1 and P2 (figure 2b) and displayed dysregulation of distinct gene sets (figures $2 c, 2 d$ ). The P1 were characterized by increased expression of genes in myeloid inflammation, type 1 interferon and innate immune signaling pathways, lower COVID antibody levels and increased T-cell receptor diversity. P2 were characterized by highly up-regulated CD44, BCL2, TGFB1, IL18BP, IL27RA, and IL11RA.

Conclusions Immune dysregulation in CCD continues at least 6 months post-infection. Some CCDs experienced marked transcriptional changes which may be the result of COVID-19 reactivation and could be responsible for long-haul syndrome.

Acknowledgements N/A

Trial Registration NCT04360278

\section{REFERENCES}

N/A

Ethics Approval N/A

Consent N/A

http://dx.doi.org/10.1136/jitc-2021-SITC2021.953 